\title{
The quality of the scientific output of 100 global leaders in the field of endocrinology
}

\author{
Wojciech Pluskiewicz', Piotr Adamczyk², Bogna Drozdzowska ${ }^{3}$, Krzysztof Noga ${ }^{4}$ \\ ${ }^{1}$ Department and Clinic of Internal Diseases, Diabetology and Nephrology, Metabolic Bone Diseases Unit, \\ Faculty of Medical Sciences in Zabrze, Medical University of Silesia, Katowice, Poland \\ ${ }^{2}$ Department of Pediatrics, Faculty of Medical Sciences in Katowice, Medical University of Silesia, Katowice, Poland \\ ${ }^{3}$ Department and Chair of Pathomorphology, Faculty of Medical Sciences in Zabrze, Medical University of Silesia, Katowice, Poland \\ ${ }^{4}$ Main Library, Medical University of Silesia, Katowice, Poland
}

W e would like to present the scientific output of 100 global leaders in endocrinology. In the current study the assessed scientific outputs, ranked by Hirsch index, were compared with figures obtained with the use of a new index: the Scientific Quality Index (SQI). Data for 100 researchers with the highest numbers of published papers were derived from the Scopus database for key words endocrine/hormone for a period of five years. The following bibliometric parameters were applied: the h-index for all citations, the h-index, calculated after the exclusion of self-citations and the citations of all co-authors, the citation index (except of the citations by the first author and by all co-authors), the number of all the published papers, the number of cited papers, the number of papers cited at least 10 times, and the percentage of papers cited at least 10 times among all the published papers, including those with no citation.

Using the selected bibliometric parameters, the SQI was calculated according to the following formula:

Parameter No. 1 + Parameter No. 2 ,

where:

- parameter No. 1 (the percentage of papers cited $\geq 10$ times) $=$ the number of papers cited $\geq 10$ times (excluding self-citations and the citations of all co-authors), divided by the number of all the published papers multiplied by $100 \%$;

- parameter No. 2 (the mean number of citations per paper) $=$ the total number of citations (excluding self-citations and the citations of all co-authors), divided by the number of all the published papers.

The following values were obtained for bibliometric variables: the number of all the published papers $-15.9 \pm 4.8$; the citation index $-150 \pm 122$; the number of cited papers $-13.2 \pm 5.1$; the number of papers cited at least 10 times $-4.7 \pm 3.2$; the percentage of papers cited at least 10 times, out of all the published papers, including those with no citation $-32.0 \pm 18.1$; the mean number of citations per paper $-10.1 \pm 8.3$; h-index: $6.44 \pm 2.1$; and SQI: $42.1 \pm 25.0$. When the analysed subjects were ranked by the h-index and SQI, the most important finding was that $96 \%$ of the authors changed their initial ranking position, established primarily by the h-index. Out of all the authors, 55 shifted upwards, while 41 shifted downwards vs. their initial h-index ranking position.

All parameters correlated significantly with the h-index, while SQI did not correlate with pure quantitative variables, e.g. either with the number of all the published papers or with the number of cited papers. Regarding other variables, SQI correlated significantly well. As indicated by the Fischer test, all the coefficients of correlation for the h-index and SQI differed significantly, 


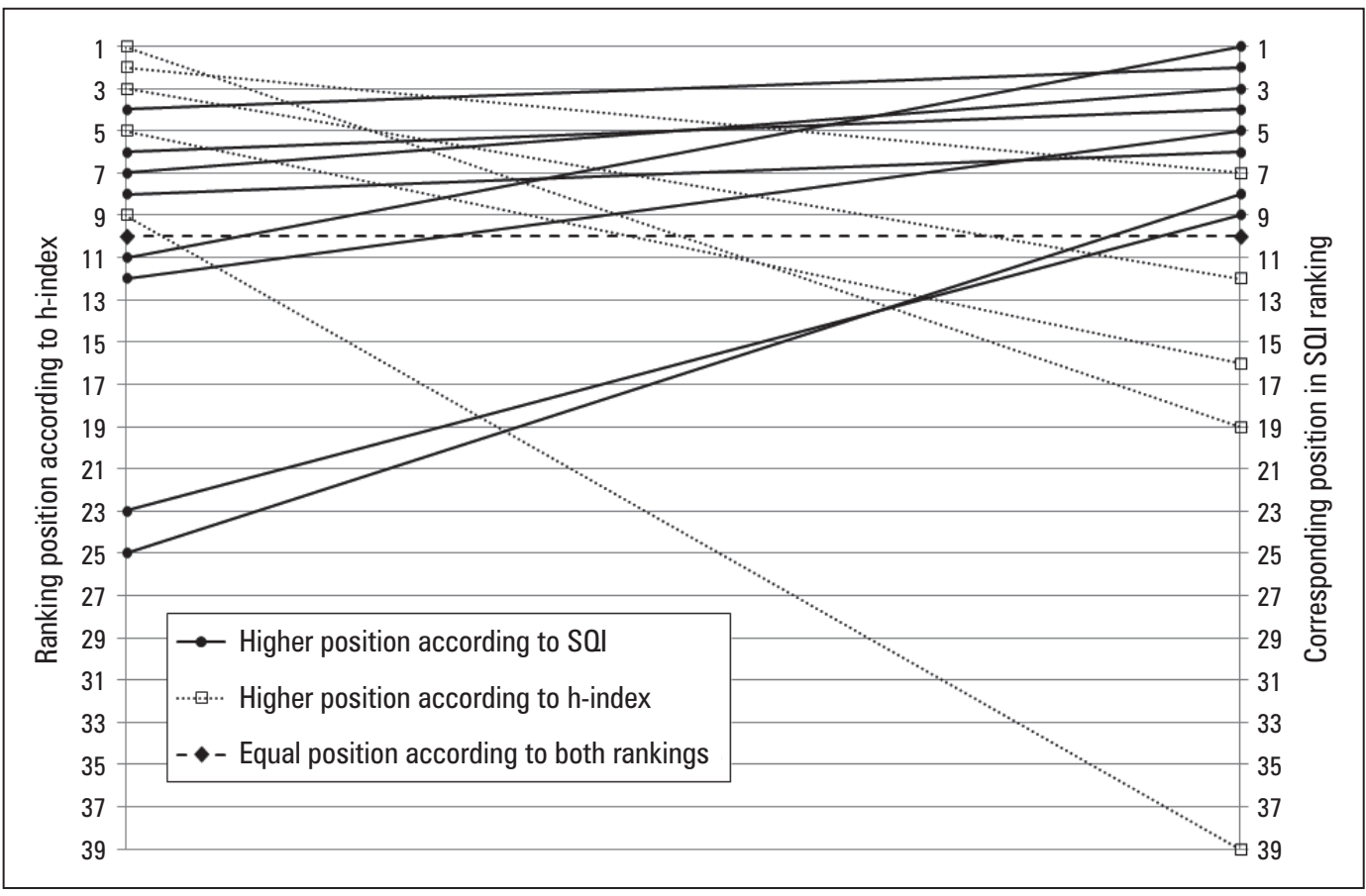

Figure 1. Corresponding ranking positions, provided by h-index and Scientific Quality Index (SQI), for the researchers rated among "the top 10" in at least one of the analysed ranking systems

Table I. Correlation analysis and comparison between $\mathbf{r}$ values for the h-index and Scientific Quality Index (SQI)

\begin{tabular}{lccc}
\hline Variable & $\begin{array}{c}\text { r value for } \\
\text { the h-index }\end{array}$ & r value for SOI & $\begin{array}{c}\text { p value according } \\
\text { to the Fischer test }\end{array}$ \\
\hline Number of publications & $0.31^{*}$ & -0.11 (NS) & $<0.01$ \\
\hline Citation index & $0.87^{*}$ & $0.85^{*}$ & $\mathrm{NS}$ \\
\hline Number of cited papers & $0.57^{*}$ & 0.16 (NS) & $<0.001$ \\
\hline Number of papers cited $\geq \mathbf{1 0}$ times) & $0.85^{*}$ & $0.93^{*}$ & $<0.01$ \\
\hline First parameter of SOI & $0.73^{*}$ & $0.99^{*}$ & $<0.001$ \\
\hline Second parameter of SOI & $0.78^{*}$ & $0.95^{*}$ & $<0.0001$ \\
\hline
\end{tabular}

${ }^{*} p<0.0001 ; N S$ - not significant

except $\mathrm{r}$ values for the citation index. The h-index and SQI correlated significantly with each other $(0.75, \mathrm{p}<0.0001)$. Figure 1 presents the corresponding ranking positions, obtained in both assessment systems (this presentation is limited to 14 researchers with positions among "the top $10^{\prime \prime}$ achieved at least in one of the ranking systems). Table I presents a correlation analysis.

Concluding, SQI is a new tool, able to pick up the qualitative features from assessed scientific output in individual authors. SQI presents the unique feature of having the possibility to decrease over time, and so it may be recommended for longitudinal assessment of scientific output quality.

\section{References}

1. Hirsch JE. An index to quantify an individual's scientific research output. Proc Natl Acad Sci USA. 2005; 102(46): 16569-16572, doi: 10.1073/pnas.0507655102, indexed in Pubmed: 16275915.

2. Pluskiewicz W, Drozdzowska B. A novel indicator of the scientific quality in academic medical researchers. CEON Repozytorium. https://depot.ceon.pl/handle/123456789/12553?show = full (16 November 2017).

3. Pluskiewicz W, Drozdzowska B, Adamczyk P, et al. Scientific output quality of 40 globally top-ranked medical researchers in the field of osteoporosis. Arch Osteoporos. 2018; 13(1): 35, doi: 10.1007/s11657-018-0446-4, indexed in Pubmed: 29582219.

4. Pluskiewicz W, Drozdzowska B, Adamczyk P, et al. Scientific Quality Index: a composite size-independent metric compared with h-index for 480 medical researchers. Scientometrics. 2019; 119(2): 1009-1016, doi: 10.1007/s11192-019-03078-z. 